\title{
统计模型的辫子群矩阵与不变量
}

赵宏康

葛墨林

(兰州大学物理系, 兰州 730000)（南开数学研究所理论物理研究室, 天津 300071)

\section{关链词样杨-Baxter 矩阵、统计輠型、拒扑不变量}

辫子群与二维统计模型有密切的联系. Akutsu 和 Wadati 把六一顶角模型及其高自旋系 列的杨-Baxter 方程解 $S(x)$ 矩阵中谱参数取无穷极限得到了 “自旋数” 守恒的辫子群表示 ${ }^{[1]}$. 对 $Z_{N}$ 模型用上述方法很难得出有意义结果, 因 $S(x)$ 矩阵是用具有双周期的椭圆函数参数化.

一般地, 杨-Baxter 方程里三个谱参数不必有 $u^{\prime \prime}-u^{\prime}-u$ 的限制, 可设 $u^{\prime \prime}-f\left(u, u^{\prime}\right)$. 如果在杨-Baxter 方程中取极限 $u, u^{\prime}, u^{\prime \prime} \rightarrow u_{0}$, 就可去掉谱参数, 便有

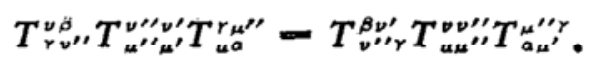

因此, 辫子群杨-Baxter 方程(1)是带谱参数杨-Baxter 方程的极限.

定义辫子群生成元

$$
g_{i}=I^{(1)} \otimes \cdots \otimes I^{(i-1)} \otimes T \otimes I^{(i+2)} \otimes \cdots \otimes I^{(n)},
$$

就可构造一个非平庸的辫子群 $B_{n}-\left(g_{1}, g_{2}, \cdots, g_{n-1}\right)$ 表示, 并得拓扑不变多项式. 所以有两 种构造辫子群表示方法：一种是解方程 (1); 另一种是使统计模型中 $S(x)$ 矩阵的谱参数趋于 某个定数.

Baxter 的八一顶角或 $Z_{2}$ 模型 $S(x)$ 矩阵的非零元为 $S_{00}^{00}-S_{11}^{4}-a, S_{10}^{10}-S_{01}^{01}=b, S_{01}^{10}=$ $S_{10}^{01}-c, S_{00}^{11}-S_{11}^{00}-d$. 代人(1)式, 有 $a= \pm b, c= \pm d$.

对于 $Z_{N}$ 模型, 由方程(1)解得 $T=P S$, 其中 $P$ 为置换算符, $S=\sum_{i=0}^{N-1} W_{i} I_{i} \otimes I_{i}^{-1}$,

$$
\left(I_{i}\right)_{\mu \nu}=\delta(\mu-\nu-i),\left(I_{i}^{-1}\right)_{\mu \nu}-\delta(\mu-\nu+i),(i, \mu, \nu=0,1, \cdots, N-1),
$$

显然 $\left[I_{i}, I_{j}\right]-0$.

$$
\delta(y)=\left\{\begin{array}{l}
1, \text { 当 } y=0, N, \\
0, \text { 当 } y=1, \cdots, N-1 .
\end{array}\right.
$$

$Z_{2}$ 的辫子群表示矩阵可用投影矩阵 $P_{i}$ 展开 $T-\sum_{i=1}^{4} \lambda_{i} P_{i}$, 其中 $\lambda_{i}(i-1, \cdots, 4)$ 是 $T$ 的本征值, $\lambda_{1}-W_{0}+W_{1}, \lambda_{2}-W_{0}-W_{1}, \lambda_{1}-\lambda_{3}, \lambda_{2}=-\lambda_{4}$.

由辫子群矩阵 $T$ 恢复出带谱参数的 $S(x)$ 矩阵

$$
S(x)=p(x) T^{-1}+q(x) I+f(x) T,
$$

这里 $\quad q(x)=\lambda_{1} \lambda_{4} \frac{\sin x}{1+\left(\lambda_{1}-1\right) \sin x}, p(x)-\frac{1}{2} \lambda_{1} \lambda_{2}\left[\lambda_{2}-\frac{\cos ^{2} x}{1+\left(\lambda_{1}-1\right) \sin x}\right]$,

$$
f(x)=-\frac{1}{2}\left[\lambda_{1}-\frac{\cos ^{2} x}{1+\left(\lambda_{1}-1\right) \sin x}\right], W_{0}=1 .
$$

谱参数 $x, x^{\prime}, x^{\prime \prime}$ 之间满足关系

$$
\sin x^{\prime}-\frac{\sin x^{\prime \prime}+\sin x}{1+\sin x^{\prime \prime} \sin x}
$$

1991-09-04 收稿, 1992-06-15 收修改稿 
在(3)式中, 当 $x \rightarrow \pi / 2+2 n \pi$ 时遂有 $S(x) \rightarrow T$. 另外, 若采取映射 $\sin x \mapsto-i \operatorname{tg} u, W_{1}$ $\mapsto-i \operatorname{tg} 2 \eta$, 则矩阵 (3) 映射为

$$
\begin{gathered}
a-i \rho \operatorname{tg}(u+2 \eta), b-i \rho \operatorname{tg} u, \\
c=i \rho \operatorname{tg} 2 \eta, d--i \rho \operatorname{tg} 2 \eta \operatorname{tg} u \operatorname{tg}(u+2 \eta) .
\end{gathered}
$$

此 $S(x)$ 矩阵与 Faddeev, Takhtadzhan 所得到的 $R(x)$ 矩阵当 $k \rightarrow 1$ 时相一致, 即矩阵(3) 是 $R(x)$ 矩阵的临界情形 ${ }^{[2]}$. 由(6)式取极限 $u \rightarrow i \infty$ 亦可得 $T$.

与 $Z_{N}$ 的辫子群相应的 Markove 迹是

$$
\phi(A)=\operatorname{Tr}(H A), A \in B_{n},
$$

其中

$$
\begin{aligned}
& H=h \otimes h \otimes \cdots \otimes h \otimes \cdots, h=Z / \operatorname{tr} Z, \\
& Z_{\alpha \beta}=\delta_{\alpha \beta},(\alpha, \beta-0,1, \cdots, N-1) .
\end{aligned}
$$

由 Markove 迹和“降阶关系”构造得 $Z_{2}$ 的 Link 多项式

$$
\alpha(A)=\left(W_{0}+W_{1}\right)^{-e(A)} \phi(A),\left(A \in B_{n}\right)
$$

和 “Alexander-Conway 关系”

$$
\alpha\left(L_{2+}\right)=\alpha\left(L_{+}\right)+\left(\frac{W_{0}-W_{1}}{W_{0}+W_{1}}\right)^{2} \alpha\left(L_{0}\right)-\left(\frac{W_{0}-W_{1}}{W_{0}+W_{1}}\right)^{2} \alpha\left(L_{-}\right) .
$$

自由参数 $W_{0}$ 和 $W_{1}$ 可以约简为一个. $e(A)$ 是 $g_{i}$ 在辫子 $A$ 中出现的指数和. $L_{2+}, L_{+}, L_{0}$ 和 $L_{-}$指对应于 $g_{i}^{2}, g_{i}, g_{i}^{0}$ 和 $g_{i}^{-1}$ 的联结.

$Z_{3}$ 的辫子群矩阵 $T=P S$, 这里

$$
S=\sum_{i=0}^{i} W, I, \otimes I_{i}^{-1}
$$

逆矩阵 $T^{-1}=P S^{-1}$, 其中 $\quad S^{-1}=\sum_{i=0}^{2} \widetilde{W}_{i} I_{i} \otimes I_{i}^{-1}$,

$$
\widetilde{W}_{0}-\frac{1}{a_{3}}\left(W_{1} W_{2}-W_{0}^{2}\right), \widetilde{W}_{1}-\frac{1}{a_{3}}\left(W_{0} W_{2}-W_{1}^{2}\right), \widetilde{W}_{2}=\frac{1}{a_{3}}\left(W_{0} W_{1}-W_{2}^{2}\right) .
$$

Link 多项式

$$
\alpha(A)=\left(-\frac{a_{1} a_{2}}{a_{3}}\right)^{-(n-1) / 2}\left(-\frac{a_{2}}{a_{1} a_{3}}\right)^{e(A) / 2} \phi(A),\left(A \in B_{n}\right)
$$

和 “Alexander-Conway 关系”

$$
\alpha\left(L_{2+}\right)-\left(-\frac{a_{1} a_{2}}{a_{3}}\right)^{1 / 2} \alpha\left(L_{+}\right)-\frac{a_{2}^{2}}{a_{1} a_{3}} \alpha\left(L_{0}\right)-\frac{a_{2}}{a_{1}}\left(-\frac{a_{2}}{a_{1} a_{3}}\right)^{1 / 2} \alpha\left(L_{-}\right),
$$

其中

$$
a_{1}=\sum_{k=0}^{2} W_{k}, a_{2}=\sum_{k=0}^{1} W_{k}^{2}-\sum_{i>k=0}^{1} W_{k} W_{i}, a_{3}-3 \prod_{k=0}^{2} W_{k}-\sum_{k=0}^{i} W_{k}^{3} .
$$

设 $W_{0}=1$, 则 3 个自由参量 $W_{0}, W_{1}, W_{2}$ 约简为 2 个. 由此构造出具有 2 参量的拓扑不变 多项式. $Z_{N}$ 模型的辫子群表示有 $N-1$ 个自由参量, 原则上可构造有 $N-1$ 个自由参量的 拓扑不变多项式.

现在讨论对称上三角 $S$ 矩阵, 其不为零的矩阵元 设 为 $S_{00}^{00}-S_{11}^{11}-W_{1}, S_{00}^{01}-S_{10}^{11}-W_{2}$, $S_{0 j}^{10}-S_{01}^{11}-W_{3}, S_{00}^{11}=W_{4}, S_{01}^{01}=S_{10}^{10}=W_{5}, S_{01}^{10}=W_{6}$. 将 $T-P S$ 代人方程(1)解得 $T_{00}^{00}=$ $T_{11}^{11}=T_{10}^{01}=T_{01}^{10}-W_{1}, T_{00}^{10}-T_{10}^{11}=W_{2}, T_{00}^{01}=T_{01}^{11}=W_{3}, T_{00}^{11}-W_{4}$. “降阶关系”为 $g_{i}=$ $W_{i}^{2} g_{i}^{-1}$, 这里 $g_{i}$ 是由(2)式定义的辫子群生成元。选择 Markove $h$ 矩阵为 


$$
h_{00}-h_{11}=\frac{1}{2}, \quad h_{01}-\frac{1}{2} b, \quad h_{10}-0,
$$

其中 $b$ 是任意常参量, 于是有拓扑不变量

及 “Alexander-Conway 关系”

$$
\alpha(A)-\left(1 / W_{1}\right)^{(\alpha)} \phi(A),\left(A \in B_{n}\right)
$$

$$
\alpha\left(L_{+}\right)-\alpha\left(L_{-}\right) .
$$

同理,对称十六-顶角模型[3]的绛子群矩阵是

$$
\begin{aligned}
& T_{00}^{00}-T_{01}^{10}-T_{10}^{11}-T_{11}^{11}-W_{1}, T_{00}^{10}-T_{01}^{00}-T_{11}^{01}-T_{10}^{11}-W_{10}, \\
& T_{00}^{01}-T_{01}^{11}-T_{10}^{00}-T_{11}^{10}-W_{3}, T_{00}^{11}-T_{01}^{01}-T_{10}^{10}=T_{11}^{00}=W_{\imath},
\end{aligned}
$$

其中 $W_{i}(i-1, \cdots, 4)$ 是任意自由参量. 可证此 $T$ 矩阵满足辫子群杨-Baxter 方程(1), 且 本征值为

$$
\begin{aligned}
& \lambda_{1}-\sum_{i=1}^{\infty} W_{i}, \lambda_{2}-W_{1}+W_{4}-W_{2}-W_{3}, \\
& \lambda_{3}-\left[\left(W_{1}-W_{4}\right)^{2}-\left(W_{2}-W_{3}\right)^{2}\right]^{1 / 2}, \lambda_{4}=-\lambda_{3} .
\end{aligned}
$$

拓扑不变 Link 多项式

$$
\alpha(A)=\left[\frac{1}{4}\left(W_{1}+W_{4}\right)^{2} \beta\right]^{-(n-1) / 2} \beta^{c(A) / 2} \phi(A),\left(A \in B_{n}\right),
$$

其中

$$
\begin{gathered}
\beta-\frac{1}{M\left(W_{i}+W_{4}\right)}\left\{W_{1} \sum_{i=1}^{1} W_{i}^{2}+W_{+} \sum_{i=1}^{1} W_{i}^{2}-2\left(\prod_{i=1}^{1} W_{i}+\prod_{i=1}^{3} W_{i}\right)-\left(W_{1}^{3}+W_{i}^{3}\right)\right\}, \\
M-2 \sum_{i=i<i}^{\dot{1}}\left(W_{i} W_{i}\right)^{2}-\sum_{i=1}^{\dot{1}} W_{i}^{4}-8 \prod_{i=1}^{+} W_{i},
\end{gathered}
$$

这里 Markove 迹中的 $h$ 为 $2 \times 2$ 单位矩阵.

“Alexander-Conway 关系”是

$$
\alpha\left(L_{3+}\right)-a_{1} \beta^{1 / 2} \alpha\left(L_{2+}\right)+a_{2} \beta \alpha\left(L_{+}\right)+a_{3} \beta^{3 / 2} \alpha\left(L_{0}\right)+a_{4} \beta^{2} \alpha\left(L_{-}\right),
$$

这里

$$
\begin{aligned}
& a_{1}=2\left(W_{1}+W_{4}\right), \quad a_{2}-4\left(W_{2} W_{3}-W_{1} W_{4}\right), \\
& a_{3}=2\left(W_{1}+W_{4}\right)\left[\left(W_{2}-W_{3}\right)^{2}-\left(W_{1}-W_{4}\right)^{2}\right], \\
& a_{4}=\left[\left(W_{1}+W_{4}\right)^{2}-\left(W_{2}+W_{3}\right)^{2}\right]\left[\left(W_{1}-W_{4}\right)^{2}-\left(W_{2}-W_{3}\right)^{2}\right] .
\end{aligned}
$$

由此可知, 直接解方程 (1) 是构造辫子群表示的有效方法; 统计模型与辫子群有必然的联 系; 通过韸子群表示便能找到其拓扑不变量.

补充说明, 将八一顶角模型的临界情形 $k \rightarrow 1$ 时 $S(u)^{[4]}$ 中谱参数 $u$ 取极限 $u \rightarrow i \infty$ 可得到 另一个辫子群表示: $W_{1}=-W_{2}=\rho, W_{3}=W_{4}=i \rho \operatorname{tg} \lambda$.

[1] Akutsu, K., Wadati, M., J. Phys. Soc. Japan, '6(1987), 3039.

[2] Takhtadzhan, L. A., Faddeev, L. D., Russian Math. Surveys, 34(1979), 11.

[3] Phase Transitions and Critical Phenomena, Vol. I, Academic Press, London, New York, 1972, MR50 E391.

[4] Baxter, R. J., Exactly Solved Models in Statistical Mechanics. Academic Press, New York, 1982. 